\title{
Prevalence of antipsychotic polypharmacy: prescribing practices at the psychiatry department at HMC (hamad medical corporation, doha, qatar)
}

\begin{abstract}
Combining antipsychotics in the treatment of schizophrenia and other psychotic disorders is relatively common. Rates in the literature have varied with an average ranging from $10 \%$ to $40 \% .{ }^{1}$ A recently published systematic review that summarized 173 studies reporting on rates and correlates of antipsychotic polypharmacy from the 1970s to today in over 1.5 million patients found that the overall mean antipsychotic polypharmacy rate was $26 \% .^{2}$ Concerns about antipsychotic polypharmacy include the possibility of higher than necessary numbers of medications, greater than necessary total dosages, increased acute and long-term adverse effects, drug-drug interactions, increased non-adherence, difficulties in determining the effect of each treatment, substantially higher cost, a risk for increased mortality, and the general lack of evidence for the effectiveness and safety of antipsychotic polypharmacy. ${ }^{3-8}$

It is our observation that despite the lack of clinical evidence, the anti-psychotic prescribing practices are very prevalent in our department. So far there was no quantitative or qualitative study that looked into this important issue. But there is little literature evidences found regarding the benefits of adding another antipsychotic. We are set to review the prescribing practices at the department of Psychiatry, HMC, in regard to the Antipsychotic polypharmacy, and see how it compares to the international general psychiatric practice then compare it to the existing guidelines. The results are discussed, recommendations are drafted and we plan on follow up in a separate yet later study the progress we would made in bringing about any change in the prescribing behavior after a remedial one year of educational intervention.
\end{abstract}

Keywords: polypharmacy, antipsychotics, treatment of psychotic disorders
Volume 3 Issue 4 - 2015

\author{
Adel Sleiman Zaraa, Majid Al-Abdulla, \\ Waseem Abdallah, Mahmoud Aborabeh, Soha \\ Mahmoud \\ University OUCOM, Ohio, USA, WCMC, Qatar
}

Correspondence: Adel Sleiman Zaraa, Professor of Clinical Psychiatry, University OUCOM, Ohio, USA, WCMC, HMC, ED Po Box 3050, Doha, Qatar, Tel 974 3342-7277,

Emaildrzaraa@gmail.com

Received: September 09, 2015 | Published: September 09, 2015

\section{Introduction}

\section{Concerns about antipsychotic polypharmacy}

Concerns about antipsychotic polypharmacy include the possibility of higher than necessary numbers of medications, greater than necessary total dosages, increased acute and long-term adverse effects, drug-drug interactions, increased non-adherence, difficulties in determining the effect of each treatment, substantially higher cost, a risk for increased mortality, and the general lack of evidence for the effectiveness and safety of antipsychotic polypharmacy. ${ }^{3-8}$

\section{Regional and geographic differences in prescribing practices}

It was important to us to look into any possible variation in prescribing practices based on regional and ethnic back ground because our department provides services to more than forty different nationalities and ethnic groups. This factor is equally valid for the prescribers as well.

Across 147 studies $(1,418,163$ participants, $82.9 \%$ diagnosed with schizophrenia IQR $=42-100 \%$, the median APP rate was $19.6 \%$ $(\mathrm{IQR}=12.9-35.0 \%)$. Most common combinations included firstgeneration antipsychotics (FGAs)+second-generation antipsychotics (SGAs) $(42.4 \%, \mathrm{IQR}=0.0-71.4 \%)$ followed by FGAs+FGAs $(19.6 \%$, $\mathrm{IQR}=0.0-100 \%)$ and SGAs + SGAs $(1.8 \%, \mathrm{IQR}=0.0-28 \%)$. APP rates were not different between decades (1970-1979:28.8\%, IQR=7.5$44 \% ; \quad 1980-1989: 17.6 \%, \quad$ IQR $=10.8-38.2 ; \quad 1990-1999: 22.0 \%$, $\mathrm{IQR}=11-40 ; 2000-2009: 19.2 \% \mathrm{IQR}=14.4-29.9, \mathrm{p}=0.78)$, but between regions, being higher in Asia and Europe than North America, and in Asia than Oceania $(\mathrm{p}<0.001)$. APP increased numerically by $34 \%$ in North America from the 1980s 12.7\%) to 2000s (17.0\%) ( $\mathrm{p}=0.94)$ and decreased significantly by $65 \%$ from $1980(55.5 \%)$ to 2000 $(19.2 \%)$ in Asia $(p=0.03)$, with non-significant changes in Europe. APP was associated with inpatient status $(\mathrm{p}<0.001)$, use of FGAs $(p<0.0001)$ and anticholinergics $(<0.001)$, schizophrenia $(p=0.01)$, less antidepressant use $(p=0.02)$, greater LAIs use $(p=0.04)$, shorter follow-up ( $\mathrm{p}=0.001)$ and cross-sectional vs. longitudinal study design $(p=0.03)$. In a meta-regression, inpatient status $(p<0.0001)$, FGA use $(0.046)$, and schizophrenia diagnosis $(p=0.004)$ independently predicted APP $(\mathrm{N}=66, \mathrm{R} 2=0.44, \mathrm{p}<0.0001)$. Antipsychotic poly pharmacy is common practice in psychiatry.

Despite that the controlled evidence for its efficacy and safety as a strategy remains inconclusive. Moreover, antipsychotic polypharmacy was more common in schizophrenia, inpatients, adults, patients treated with decanoate formulations and with anticholinergics, and in patients treated in Asia. The number of studies reporting on rates of antipsychotic polypharmacy is in stark contrast to investigations of prescriber reasons and patient responses, data that are needed to inform clinical practice.

\section{Tolerability of antipsychotic polypharmacy}

There are relatively consistent reports of higher antipsychotic doses in patients receiving antipsychotic polypharmacy. ${ }^{6}$ Therefore, it is not surprising that antipsychotic polypharmacy has been associated with dose-related side effects at the dopamine receptor, such as hyperprolactinemia9,10 and extrapyramidal side effects that were reported either directly, ${ }^{11}$ or indirectly, expressed as hyper- 
salivation..$^{10,12}$ or an increased use of anticholinergics. ${ }^{13,14}$ Other than that, reports about the tolerability of antipsychotic polypharmacy are limited and mostly inconclusive. In isolated reports, antipsychotic polypharmacy has been associated with increased side effects in general, ${ }^{15,16}$ sedation, ${ }^{9}$ cognitive problems, ${ }^{17}$ weight gain, ${ }^{18}$ dyslipidemia, ${ }^{19}$ glucose elevation and diabetes mellitus, ${ }^{20,21}$ metabolic syndrome, ${ }^{19}$ and cardiovascular death. ${ }^{22,23}$

\section{Methods}

In this study, the authors are set to review the prescribing practices at the department of Psychiatry, in regard to the Antipsychotic polypharmacy, and see how it compares to the international general psychiatric practice then compare it to the existing guidelines. This is a retrospective descriptive study based on electronic charts review of all admissions to the psychiatry department during a six month period of 2012; among other parameters, we have analyzed them for prescribing patterns related to polypharmacy. We recorded the diagnosis, number of prescribed anti psychotics and doses, age, sex, ethnicity and the length of stay of the patients admitted in that period. The data was collected anonymously, pooled and then studied, and analyzed using SPSS 21 statistical software program. All admissions were included and there were no exclusion criteria.

Table I That amounted to a total of 527 patients. Compared to the Qatari population, the distribution of gender was slightly skewed toward the number of female patients compared to male patients, but that was expected because globally

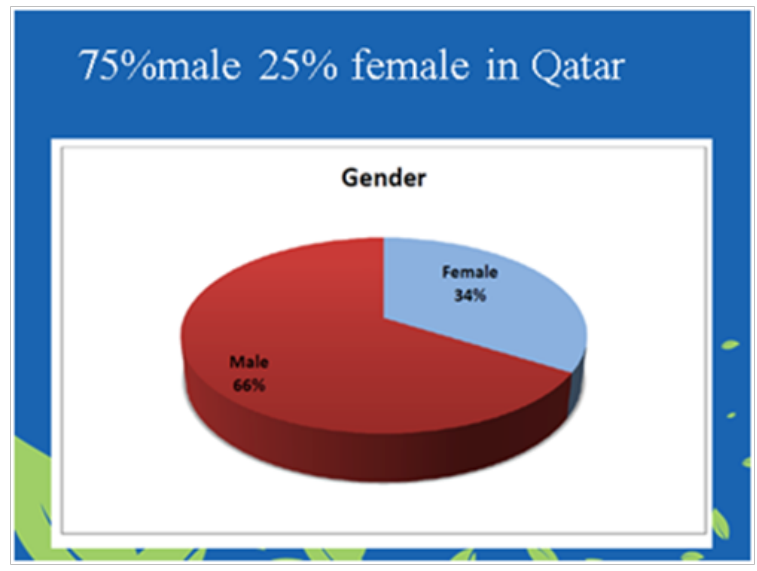

Table 2 The pyramid of population living in Qatar does not follow a normal pattern, where we have I female for every 3 males, and the expat working population between the ages of 20 and 45 represents the majority in both sexes

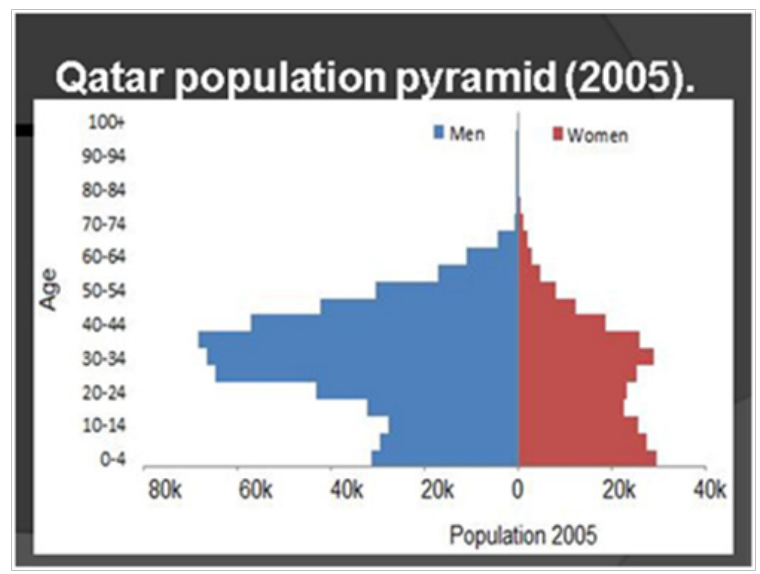

This is also congruent and reflective of our patients

\section{Results}

The review of 527 patient files, $33.8 \%$ female and $66.2 \%$ males (Table 1) who originated from 48 different nationalities (Table 2) with age ranging from 13years old to 77 years old and an average length of stay of 15.5 days were admitted to psychiatry (Table 3 ). The distribution of admitting diagnosis was peculiar for having a rather large representation of Acute and transient Psychosis mainly among the newly arrived guest workers, also that the number of bipolar disorder patients hospitalized surpassed those with schizophrenia (Table 4). 433 or $82 \%$ were prescribed antipsychotics. 241 patients or $55.6 \%$ received one antipsychotic (Table 5). While 192 patients a $44.4 \%$ received polypharmacy; of those patients $59.3 \%$ (114) received two antipsychotics (Table 6), (61) a 31.8\% were given three antipsychotics). Most of those who were taking 3 antipsychotics were diagnosed as schizophrenic, being at twice the number of patients with bipolar diagnosis. The patients with acute and transient psychosis continued to be highly represented at $17 \%$ of the sample. 17 patients, an $8.9 \%$ were treated with four simultaneous antipsychotics. Here, we looked at the dosage of drugs prescribed, and in every case, each drug was in small, subtherapeutic doses(Table 7).

Table 3 Qatar is a host to over 50 nationalities, representing different percentages of the total population.

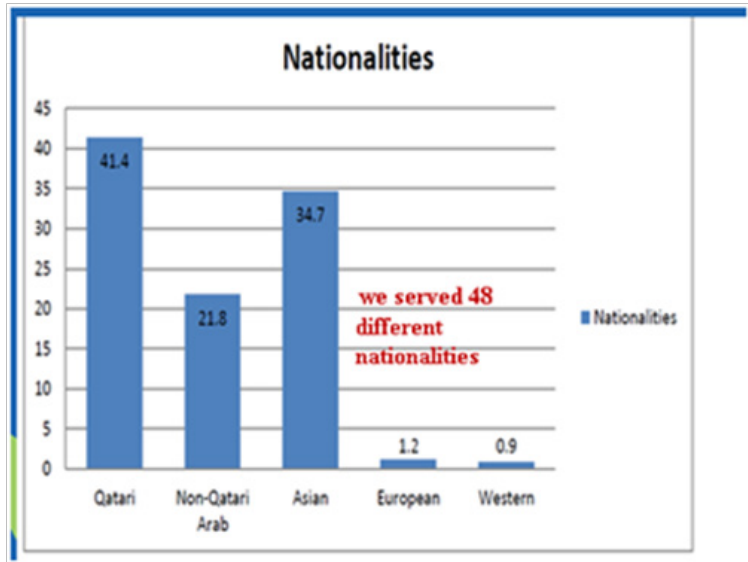

During the six months of review, we counted a service rendered to 48 different nationalities, being that the Qatari population was represented by over $41 \%$ of all inpatient services, while it represents only about $20 \%$ of the existing population

Table 4 The frequency of admitting diagnosis shows more Bipolar disorder patients admitted at a rate of $33 \%$ compared to schizophrenia at $23 \%$. It was noticeable that the diagnosis of acute and transient psychosis reaches $15 \%$ of admissions, mostly with recently-arriving guest workers

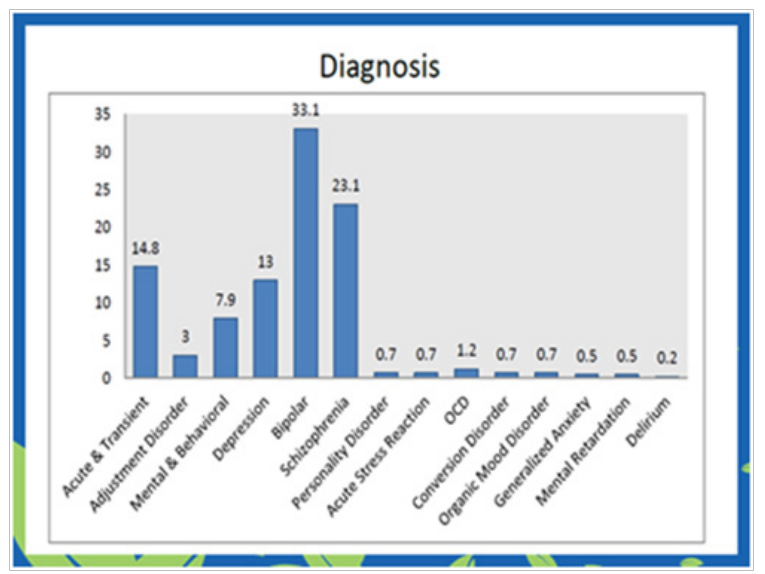

Citation: Zaraa AS,Al-Abdulla M,Abdallah W, et al. Prevalence of antipsychotic polypharmacy: prescribing practices at the psychiatry department at HMC (hamad medical corporation, doha, qatar).J Psychol Clin Psychiatry. 20 I 5;3(4): I I-I 2. DOI: I0.15406/jpcpy.20I5.03.00 I40 
Table 5 Out of the 527 patients who were admitted, 433 patients were on some kind of antipsychotic, which represented $82 \%$ of all admitted patients. Of those patients, $55 \%$ were prescribed one antipsychotic and 192 patients, being $44 \%$, were on some kind of antipsychotic polypharmacy

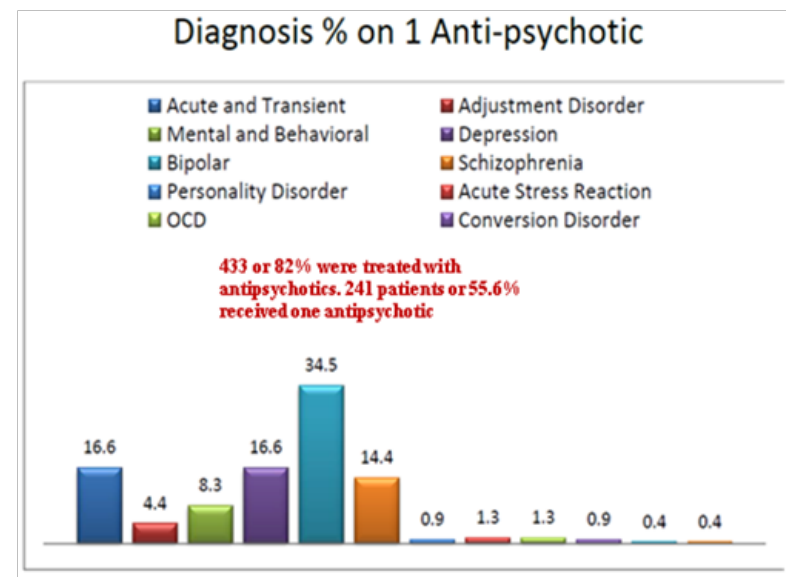

Table 6 The trend continues with almost 30\% of all patients receiving two antipsychotics.And here again, the majority of those patients were diagnosed as bipolar disorder, followed by schizophrenia, then acute and transient psychosis

\section{Diagnosis on 2 Medications}

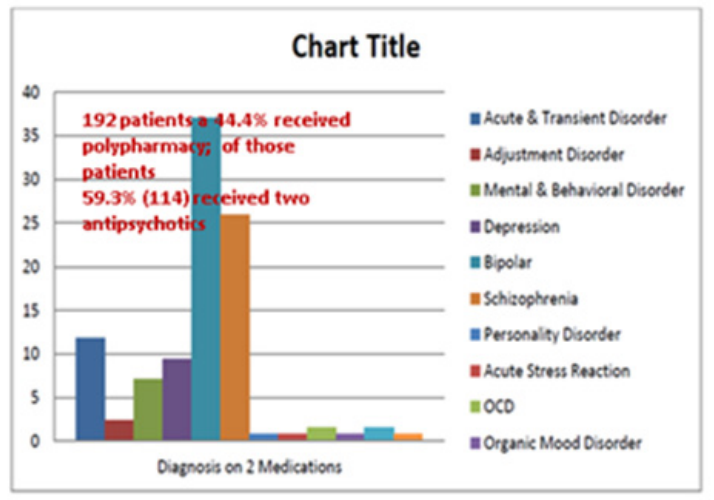

Table 7 Furthermore, 17 patients, representing 8.9\%, were on four different antipsychotics, where schizophrenia represented almost half, or 7 patients. Here, we looked at the dosage of drugs prescribed, and in every case, each drug was in small, subtherapeutic doses

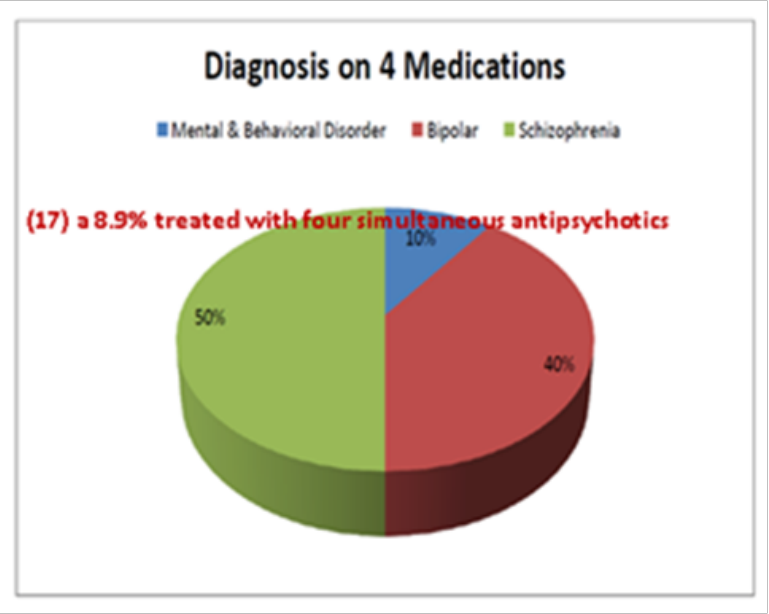

\section{Discussion}

It is clear that we do have a problem. The prevalence of antipsychotic polypharmacy in our hospital is by far much higher than the commonly found trend in hospitals across the world where the overall mean antipsychotic polypharmacy rate was $26 \% ;{ }^{2}$ despite all evidencebased medicine and guidelines that do not support a systematic practice of polypharmacy and no benefit from adding another antipsychotic before reaching therapeutic level for a therapeutic length of time with the first antipsychotic, that was another trend that is not supported by literature where a second and even third anti-psychotic was prescribed before reaching a recommended and therapeutic dosage and level on the first prescribed substance, thus many patients ended receiving two, three or four subtherapeutic dosages of anti-psychotics which added the burden of cumulative side effects without any proven clinical benefit. We made a conscious effort not to look into individual practitioners prescribing habits for our goal was to find what benefits our patients.

The impact of anti-psychotic polypharmacy has been associated with increased morbid obesity, increased diabetes mellitus and hyperlipidemia as well as increased movement disorders and cardiac morbidity. By shedding the light on poly pharmacy practices we hope to raise the awareness of prescribers regarding the many added side effects that inflicts the patients receiving these drugs: Metabolic syndrome (Diabetes and Hyper-lipidimiea), obesity, Hypertension, increased extra pyramidal symptoms and Tardive Dyskinesia, increased cardiac morbidity.

\section{Recommendations}

Our aim is to use these results to raise the awareness of the prescribers about the Evidence Based Medicine and the guidelines regarding polypharmacy. One of the first steps would be implementing cyclical seminars for all psychiatric staff to educate those regarding accepted practices and approved guidelines when it comes to prescribing and monitoring antipsychotics; then, a year later, reaudit a similar number of admissions so we can measure outcome. To appoint a utilization review specialist and or a clinical pharmacist who would flag a file with polypharmacy that is lacking justifying documentation right at the start of the prescription.

Further research and audits to the level of monitoring the psychiatric patients for metabolic syndrome, cardiac changes are needed to continue to improve patient health and treatment outcome. An incidental finding was the prevalence of acute and transient psychosis which is a matter with limited data in the literature. It could be the subject of another and separate review of the profile of patients admitted or discharged with that diagnosis.

\section{Acknowledgments}

None.

\section{Conflicts of interest}

Author declares there are no conflicts of interest.

\section{Funding}

None.

\section{References}

1. Correll CU, Frederickson AM, Kane JM, et al. Does antipsychotic polypharmacy increase the risk for metabolic syndrome? Schizophr Res. 2007;89(1-3):91-100. 
2. Stahl SM. Antipsychotic polypharmacy: squandering precious resources? J Clin Psychiatry . 2002;63(2):93-94.

3. Correll CU. Antipsychotic polypharmacy, part 1: Shotgun approach or targeted cotreatment? J Clin Psychiatry . 2008;69(4):674-675.

4. Stahl SM, Grady MM. A critical review of atypical antipsychotic utilization: comparing monotherapy with polypharmacy and augmentation. Curr Med Chem. 2004;11(3):313-327.

5. Stahl SM. Antipsychotic polypharmacy: squandering precious resources? J Clin Psychiatry. 2002;63(2):93-94.

6. Weiden PJ, Casey DE. "Polypharmacy": Combining antipsychotic medications in the treatment of schizophrenia. J Pract Psych Behav Health. 1999;5:229-233.

7. Meltzer HY, Kostakoglu AE. Combining antipsychotics: is there evidence for efficacy? Psychiatr Times. 2000;17:25-34.

8. Faries D, Ascher-Svanum H, Zhu B, et al. Antipsychotic monotherapy and polypharmacy in the naturalistic treatment of schizophrenia with atypical antipsychotics. BMC Psychiatry . 2005;5:26

9. Anil Yağcioğlu AE, Kivircik Akdede BB, Turgut TI, et al. A doubleblind controlled study of adjunctive treatment with risperidone in schizophrenic patients partially responsive to clozapine: efficacy and safety. J Clin Psychiatry. 2005;66(1):63-72.

10. Shiloh R, Zemishlany Z, Aizenberg D, et al. Sulpiride augmentation in people with schizophrenia partially responsive to clozapine. A doubleblind, placebo-controlled study.Br J Psychiatry. 1997;171:569-573.

11. Xiang YT, Weng YZ, Leung CM, et al. Exploring the clinical and social determinants of prescribing anticholinergic medication for Chinese patients with schizophrenia. Hum Psychopharmacol. 2007;22(3):173-180.

12. Henderson DC, Goff DC. Risperidone as an adjunct to clozapine therapy in chronic schizophrenics. J Clin Psychiatry. 1996;57(9):395-397.

13. KreyenbuhlJA, Valenstein M, McCarthy JF, etal. Long-term antipsychotic polypharmacy in the VA health system: patient characteristics and treatment patterns. Psychiatr Serv. 2007;58(4):489-495.
14. Ganguly R, Kotzan JA, Miller LS, et al. Prevalence, trends, and factors associated with antipsychotic polypharmacy among Medicaideligible schizophrenia patients, 1998-2000. J Clin Psychiatry. 2004;65(10):1377-1388

15. Barbui C, Nosè M, Mazzi MA, et al. Persistence with polypharmacy and excessive dosing in patients with schizophrenia treated in four European countries. Int Clin Psychopharmacol . 2006;21(6):355-362.

16. Centorrino F, Goren JL, Hennen J, et al. Multiple versus single antipsychotic agents for hospitalized psychiatric patients: case-control study of risks versus benefits. Am J Psychiatry. 2004; 161:700-706.

17. Elie D, Poirier M, Chianetta J, et al. Cognitive effects of antipsychotic dosage and polypharmacy: a study with the BACS in patients with schizophrenia and schizoaffective disorder. J Psychopharmacol 2009;24(7):1037-1044.

18. Centorrino F, Cincotta SL, Talamo A, et al. Hospital use of antipsychotic drugs: polytherapy. Compr Psychiatry . 2008;49(1):65-69.

19. Tirupati S, Chua LE. Obesity and metabolic syndrome in a psychiatric rehabilitation service.Aust NZ J Psychiatry. 2007;41(7):606-610.

20. Honer WG, Thornton AE, Chen EY, et al. Clozapine and Risperidone Enhancement (CARE) Study Group. Clozapine alone versus clozapine and risperidone with refractory schizophrenia. $N$ Engl J Med. 2006;354(5):472-482.

21. Citrome L, Jaffe A, Levine J, et al. Relationship between antipsychotic medication treatment and new cases of diabetes among psychiatric inpatients. Psychiatr Serv. 2004;55(9):1006-1013.

22. Joukamaa M, Heliövaara M, Knekt P, et al. Schizophrenia, neuroleptic medication and mortality. Br J Psychiatry. 2006;188:122-127.

23. Waddington JL, Youssef HA, Kinsella A. Mortality in schizophrenia. Antipsychotic polypharmacy and absence of adjunctive anticholinergics over the course of a 10-year prospective study. Br $J$ Psychiatry. 1998;173:325-329. 